\title{
Mesenchymal stem cells derived from normal gingival tissue inhibit the proliferation of oral cancer cells in vitro and in vivo
}

\author{
XIAOLI $\mathrm{JI}^{1}$, ZHIHUI ZHANG ${ }^{2}$, YING HAN ${ }^{1}$, JIANGYUAN SONG ${ }^{3}$, XIANGLIANG XU ${ }^{4}$, \\ JIANQIU JIN $^{1}$, SHA SU ${ }^{1}$, DONGDONG MU ${ }^{1}$, XIAODAN LIU ${ }^{1}$, SI XU ${ }^{1}$, HONGWEI CUI ${ }^{1}$, \\ ZHONGFANG ZHAO ${ }^{1}$, YIXIANG WANG ${ }^{5}$ and HONGWEI LIU ${ }^{1}$
}

\author{
${ }^{1}$ Department of Oral Medicine, Peking University School and Hospital of Stomatology, Haidian, Beijing 100081; \\ ${ }^{2}$ Department of Stomatology, Peking University Third Hospital, Haidian, Beijing 100191; ${ }^{3}$ Department of Stomatology, \\ Wuhan Union Hospital, Wuhan, Hubei 430022; ${ }^{4}$ Department of Oral and Maxillofacial Surgery, ${ }^{5}$ Central Laboratory, \\ Peking University School and Hospital of Stomatology, Haidian, Beijing 100081, P.R. China
}

Received July 22, 2016; Accepted September 23, 2016

DOI: $10.3892 /$ ijo.2016.3715

\begin{abstract}
The interplay between tumor cells and mesenchymal stem cells (MSCs) within tumor microenvironment plays a significant role in tumor development, and thus might be exploited for therapeutic intervention. In this study, we isolated MSCs from normal gingival tissue (GMSCs), and detected the effect of GMSCs on oral cancer cells via direct co-culture and indirect co-culture systems. The cell proliferation assay of direct co-culture showed that GMSCs could inhibit the growth of oral cancer cells. Conditioned medium derived from GMSCs (GMSCs-CM) also exerted an anticancer effect, which indicates that soluble factors in GMSCs-CM played a dominant role in GMSCs-induced cancer cell growth inhibition. To investigate the mechanism, we performed apoptosis assay by flow cytometry, and confirmed that cancer cell apoptosis induced by GMSCs could be a reason for the effect of GMSCs on the growth of oral cancer cells. Western blotting also confirmed that GMSCs could upregulate expression of pro-apoptotic genes including p-JNK, cleaved PARP, cleaved caspase-3, Bax expression and downregulate proliferation- and anti-apoptosis-related gene expression such as p-ERK1/2, Bcl-2, CDK4, cyclin D1, PCNA and survivin. Importantly, the inhibitory effect of GMSCs on cancer cells can partially be restored by blockade of JNK pathway. Moreover, animal
\end{abstract}

Correspondence to: Dr Yixiang Wang, Central Laboratory, Peking University School and Hospital of Stomatology, 22 Zhongguancun South Avenue, Haidian, Beijing 100081, P.R. China

E-mail: kqwangyx@bjmu.edu.cn

Dr Hongwei Liu, Department of Oral Medicine, Peking University School and Hospital of Stomatology, 22 Zhongguancun South Avenue, Haidian, Beijing 100081, P.R. China

E-mail: hongwei15362@163.com

Key words: mesenchymal stem cells derived from normal gingival tissue, oral cancer cell, proliferation, apoptosis, JNK pathway studies showed that GMSCs exerted an anticancer effect after oral cancer cells and GMSCs were co-injected with oral cancer cells. Taken together, our data suggest that GMSCs can suppress oral cancer cell growth in vitro and in vivo via altering the surrounding microenvironment of oral cancer cells, which indicates that GMSCs have a potential use in the management of oral dysplasia and oral cancer in future.

\section{Introduction}

Mesenchymal stem cells (MSCs) have received great interest due to multipotential differentiation, immunomodulation abilities and great potential for clinical application (1). MSCs have been used for tissue regeneration, immuno-disease treatment, and in most cases with favorable results. In recent years, the potential of using MSCs for cancer management were investigated. However, the results were not consistent. For example, adipose tissue-derived mesenchymal stem cells (ASCs) can suppress the growth of human breast cancer cell line MCF-7 via secreting IFN- $\beta$ (2) and human immortalized myelogenous leukemia line K562 by secreting DKK-1 (3). However, ASCs can promote human melanoma cell growth (4). Song et al showed that bone marrow-derived MSCs (BMSCs) could inhibit leukemia/lymphoma cell proliferation (5). However, Huang et al reported that MSCs derived from bone marrow could promote the growth of human colorectal cancer cells (6).

Why are the above results regarding MSCs treatment different? Our deduction is that the different tissue origin of MSCs and tumor cells may cause the different effects of MSCs on tumor cells. To test our hypothesis, we designed the experiments by using both MSCs and tumor cell-derived from the same region, the oral cavity. Gingival tissue is easy to obtain during tooth extraction or periodontal treatments and normally the removed gingival tissue is considered as biomedical waste $(7,8)$. Additionally, many reports have reported that MSCs can be isolated from gingival tissue (hereafter called MSCs derived from normal gingival tissue, GMSCs) (9). Compared with ASCs and BMSCs, GMSCs have 
stable phenotype, proliferate faster and are not tumorigenic $(10,11)$. Oral squamous cell carcinoma is a common cancer type in oral cancer, which accounts for $\sim 90 \%$ of oral cancer (12). Therefore, in this study, we used two oral cancer cell lines (CAL27 and WSU-HN6) as our models to investigate the effect of GMSCs on the proliferation of oral cancer cells and the underlying mechanisms involved through the co-culture of GMSCs/oral cancer cells, MTT [3-(4, 5-dimethyl-thiazol2-yl)-2,5-diphenyltetrazolium bromide], flow cytometry, cytokine analysis, western blotting, pathway inhibition assays and an animal study. Our data demonstrated that GMSCs could suppress the growth of oral cancer cells in vitro and in vivo, which indicated that GMSCs had a potential for the management of oral epithelial dysplasia and oral cancer treatment in the future.

\section{Materials and methods}

Cell lines. Human oral cancer cell lines CAL27 and WSU-HN6 were cultured in Dulbecco's modified Eagle's medium (DMEM; Gibco, Invitrogen, Carlsbad, CA, USA) supplemented with $10 \%$ fetal bovine serum (FBS; Gibco) and $1 \%$ penicillin-streptomycin in a humidified incubator at $37^{\circ} \mathrm{C}$ with $5 \% \mathrm{CO}_{2}$. Cells growing exponentially (log phase) were used in the following experiments.

Isolation of GMSCs. GMSCs were isolated and identified according to a previous study $(9,11)$. Briefly, normal gingival tissues were obtained from three donors (no. 1: male, age 29; no. 2: female, age 28; no. 3: female, age 30) with informed consent. Samples were respectively cut to small size for tissue culture and cultured in T25 flasks with $\alpha$-minimum essential medium ( $\alpha$-MEM; Gibco) containing 10\% fetal bovine serum (FBS; Gibco) and 1\% penicillin-streptomycin (hereafter referred to as complete medium), at $37^{\circ} \mathrm{C}$ in a humidified $5 \%$ $\mathrm{CO}_{2}$ atmosphere to generate GMSCs. The cultured GMSCs at passage 3-7 were used for the following experiments. The study was approved by the Ethics Committee of the School of Stomatology, Peking University (LA2014103).

Identification of GMSCs. GMSCs were incubated with either FITC-conjugated human CD90, CD73, CD146, CD34, CD29 (Biolegend, San Diego, CA, USA), or PE-conjugated human STRO-1, CD105 (BD Biosciences, San Jose, CA, USA), CD45 antibody (Biolegend). Isotype-matched control $\mathrm{IgG}$ or $\operatorname{IgM}$ (Southern Biotechnology Associates, Birmingham, AL, USA) were used as controls. Flow cytometry was performed using Epics XL (Beckman-Coulter Inc., Fullerton, CA, USA).

The osteogenic and adipogenic differentiations of GMSCs were analyzed through the osteogenic and adipogenic induction media described previously (13). For osteogenic assay, GMSCs were cultured in $\alpha$-MEM medium supplemented with $10 \mathrm{nM}$ dexamethasone, $10 \mathrm{mM} \beta$-glycerophosphate, $0.1 \mathrm{mM}$ L-ascorbic acid-2-phosphate, and $2 \mathrm{mM}$ glutamine (Sigma-Aldrich, St. Louis, MO, USA) for 28 days. Mineralization capacities of GMSCs were detected by alizarin red $\mathrm{S}$ ( $\mathrm{pH}$ 4.2; Sigma-Aldrich) staining. For adipogenic assay, GMSCs were cultured in $\alpha$-MEM supplemented with $1 \mu \mathrm{M}$ dexamethasone, $0.5 \mathrm{mM}$ 3-isobutyl-ethylxanthine, $10 \mathrm{mg} / \mathrm{ml}$ insulin, $60 \mathrm{mM}$ indomethacin, and $2 \mathrm{mM}$ glutamine for 21 days
Adipogenic abilities were detected by lipid droplets by oil red O (Sigma-Aldrich).

GMSC/oral cancer cell direct contact co-culture assay. To investigate the effect of GMSCs on the growth of oral cancer cell lines, we used green fluorescent protein (GFP)-labeled oral cancer cells. To generate green fluorescent protein (GFP)labeled oral cancer cells, CAL27 and WSU-HN6 were infected with retrovirus carrying GFP expression cassette for 2 days to ensure that GFP was expressed in all the cancer cells. Then we co-cultured GMSCs-GFP-labeled oral cancer cells in a cellcell direct contact manner. At the indicated time points, the cell morphology was photographed and cell proliferation was evaluated by MTT assay at the indicated time points. Briefly, tumor cells $\left(1.5 \times 10^{3}\right)$ were plated in triplicate in a 96-well plate in a cell-cell direct contact manner with $0.1 \mathrm{ml}$ complete medium containing $2 \times 10^{3}, 4 \times 10^{3}, 8 \times 10^{3}$, and $1.6 \times 10^{4}$ GMSCs (the ratio of tumor:GMSCs was 3:2, 3:4, 3:8 and 3:16 (here referred to as $3: 2,3: 4,3: 8$ and $3: 16$, respectively), for the indicated times. The cell viability was measured using MTT [3-(4, 5-dimethyl-thiazol-2-yl)-2,5-diphenyltetrazolium bromide] assay. The co-culture of GMSCs and non-GFP labeled CAL-27/WSU-HN6 was used to confirm the co-culture results of GMSCs and GFP labeled CAL-27/WSU-HN6. The effect of co-culture of GMSC-tumor cells was determined by the ratio of $\mathrm{OD}_{570}$ (tumor cells and GMSCs co-culture) / [OD 570 (tumor cells alone $)+\mathrm{OD}_{570}(\mathrm{GMSCs}$ alone)]. Tumor cells alone served as controls.

Transwell co-culture assay. Oral cancer cells and GMSCs were maintained at the above ratio (the ratio of tumor:GMSCs referred to as 3:2, 3:4, 3:8 and 3:16) were plated in a 24-well plate at the same time. GMSCs were seeded in the inserts with a pore size of 24-well Transwell plate (Corning, Tewksbury, MA, USA). Tumor cells were plated in the lower Transwell chambers. GMSCs and tumor cells were in the same co-culture system via porous Transwell membrane. The cell viability was measured using MTT assay at the indicated time points. All experiments were in triplicate and repeated at least 2 times.

Generation of conditioned medium from GMSCs. GMSCs were plated at the above ratio of GMSCs and complete medium in a $10-\mathrm{cm}$ plate for the indicated times to generate conditioned media (equivalent to the ratio of tumor:GMSCs 3:2, 3:4, 3:8 and 3:16, respectively). The conditioned media were passed through $0.2 \mu \mathrm{m}$ filter and stored at $-80^{\circ} \mathrm{C}$ until use.

Conditioned medium assay. Tumor cells were plated in triplicate in a 96-well plate with the indicated conditioned medium derived from GMSCs. At the indicated time points, MTT assay was performed to determine cell viability. The growth rate was calculated with the following formula: growth rate $(\%)=\mathrm{OD}_{570}(\mathrm{Test}) / \mathrm{OD}_{570}(\mathrm{Ctrl}) \times 100 \%$.

Serum exhausted exclusive assay. To eliminate the effect of consumption of serum in conditioned medium, additional fresh serum $(0,2,5$ and $10 \%$, was added into the conditioned medium (the ratio of tumor:GMSCs was 3:16). The modified conditioned medium was used to treat tumor cells for indicate times, and cell viability was carried out using MTT assay. 
A
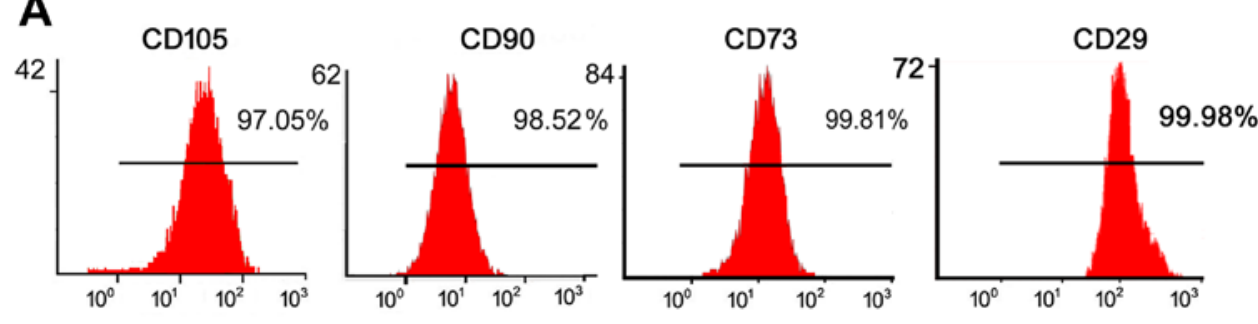

CD146

STRO-1

CD34

CD45
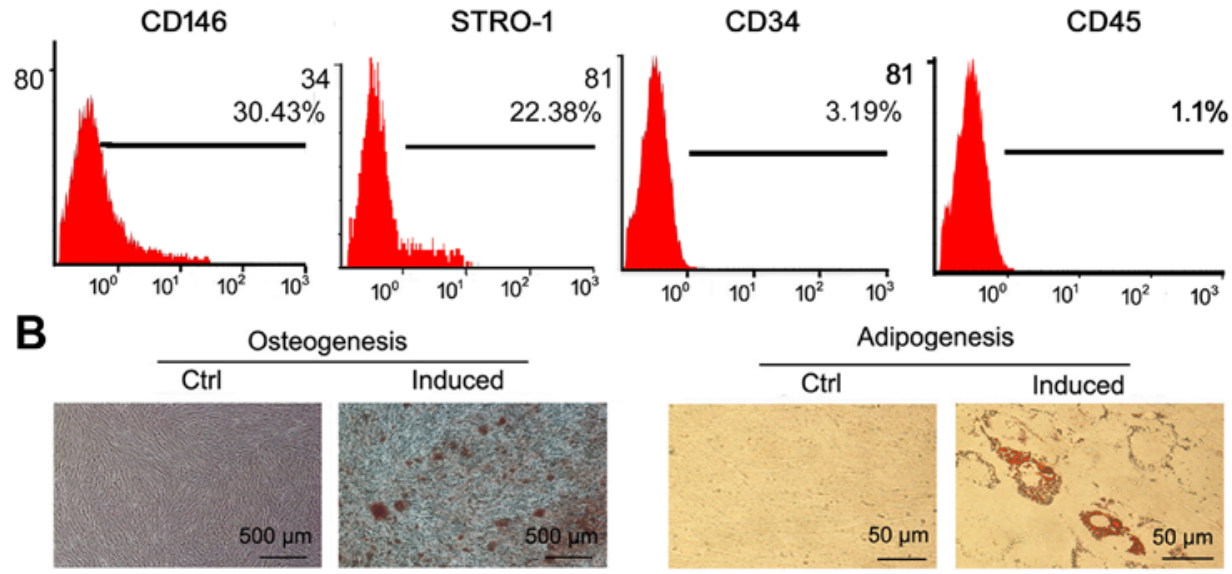

Figure 1. Identification of GMSCs. (A) Detection of the MSC surface biomarkers (CD105, CD90, CD73, CD146, STRO-1, CD29, CD34, CD45) in GMSCs by flow cytometry analysis. (B) Osteogenesis (bar, $500 \mu \mathrm{m}$ ) and adipogenesis (bar, $50 \mu \mathrm{m}$ ) of GMSCs. Under a microscope, no mineralized nodule or lipid droplet was found in the corresponding control groups, respectively.

Detection of apoptosis by flow cytometry. Conditioned medium was harvested from GMSCs in a $10-\mathrm{cm}$ plate cultured (equivalent to the ratio of tumor:GMSCs was 3:4) for 5 days. CAL27 and WSU-HN6 cells were treated for $48 \mathrm{~h}$ with above conditioned medium. Then the cells were labeled with Annexin V-FITC/PI staining according to the manufacturer's instructions (Beyotime, Shanghai, China), and subjected to do apoptotic analysis by flow cytometry (Beckman-Coulter Inc., Brea, CA, USA).

Determination of cytokine concentration in conditioned medium by cytokine array. Conditioned medium was prepared as described above, and then harvested from GMSCs (equivalent to the ratio of tumor:GMSCs was 3:4) at day 5. Then the media were used to detect multiple cytokine expression levels that allows for a detection of 9 different cytokines, respectively (Millipore Corp., Billerica, MA, USA) according to the manufacturer's protocol. Measurements were performed on Luminex 100 System (Luminex Corp., Austin, TX, USA).

Effects of reconstruction of conditioned medium on oral cancer cell growth. Based on the cytokine array results, the same concentration of multiple cytokines were chosen to mix together in various combinations to mimic the conditioned medium released from GMSCs. Then the reconstructed conditioned media were used to treat tumor cells, cell viability was detected by MTT assay as described above.

Western blotting. CAL27 and WSU-HN6 were treated with 5-day conditioned media (equivalent to the ratio of tumor:GMSCs was 3:4) for 24 or $48 \mathrm{~h}$. The cells were harvested for protein extraction at indicated time points. Protein concentration was tested using the BCA protein assay (Thermo Fisher Scientific). Equal amount of proteins were separated by $10 \%$ sodium dodecyl sulfate polyacrylamide gel electrophoresis, and then transferred to polyvinylidene difluoride membranes. The membranes were blocked in 5\% non-fat dry milk for $1 \mathrm{~h}$ and probed with antibodies against Bax, Bcl-2, cleaved caspase-3, PARP, phosphorylated (p)-STAT3, total (t)-STAT3, p-ERK1/2, t-ERK1/2, t-JNK, cyclin D1, CDK4, survivin, PCNA (Cell Signaling Technology, Danvers, MA, USA), p-JNK (Abcam, Cambridge, MA, USA), and GAPDH (Santa Cruz Biotechnology, Santa Cruz, CA, USA), respectively at $4^{\circ} \mathrm{C}$, overnight. On the following day, the protein blots were incubated with HRP-linked secondary antibody. Immunoreactive proteins were visualized by enhanced chemiluminescence (ECL) reagent (Applygen Technology, Beijing, China).

Pathway inhibition assay. Tumor cells were pre-treated with JNK inhibitor SP600125 (10 nM) (Selleck, Houston, TX, USA) for $1 \mathrm{~h}$, then the medium was replaced by conditioned medium with $10 \mathrm{nM} \mathrm{JNK}$ inhibitor SP600125 at the indicated times for MTT and western blot assays.

Animal experiment. This study was approved by Medical Ethics Committee of the Peking University Health Center (LA2014-103). Six-week-old male BALB/c nude mice (Vital River Laboratory Animal Technology Co., Ltd., Beijing, China) were divided into two groups: control group, $2 \times 10^{6}$ CAL27 in $100 \mu 1$ PBS were injected subcutaneously (s.c.) at the dorsal back of nude mice; treatment group: $2 \times 10^{6}$ CAL27 and $1 \times 10^{6}$ GMSCs in $100 \mu \mathrm{l}$ PBS were co-injected s.c. at the dorsal of nude mice. The tumor size was measured every 4 days. Tumor size was measured by caliper, and tumor volume was calculated according to formula: volume = length $\mathrm{x}$ width ${ }^{2} / 2$. 


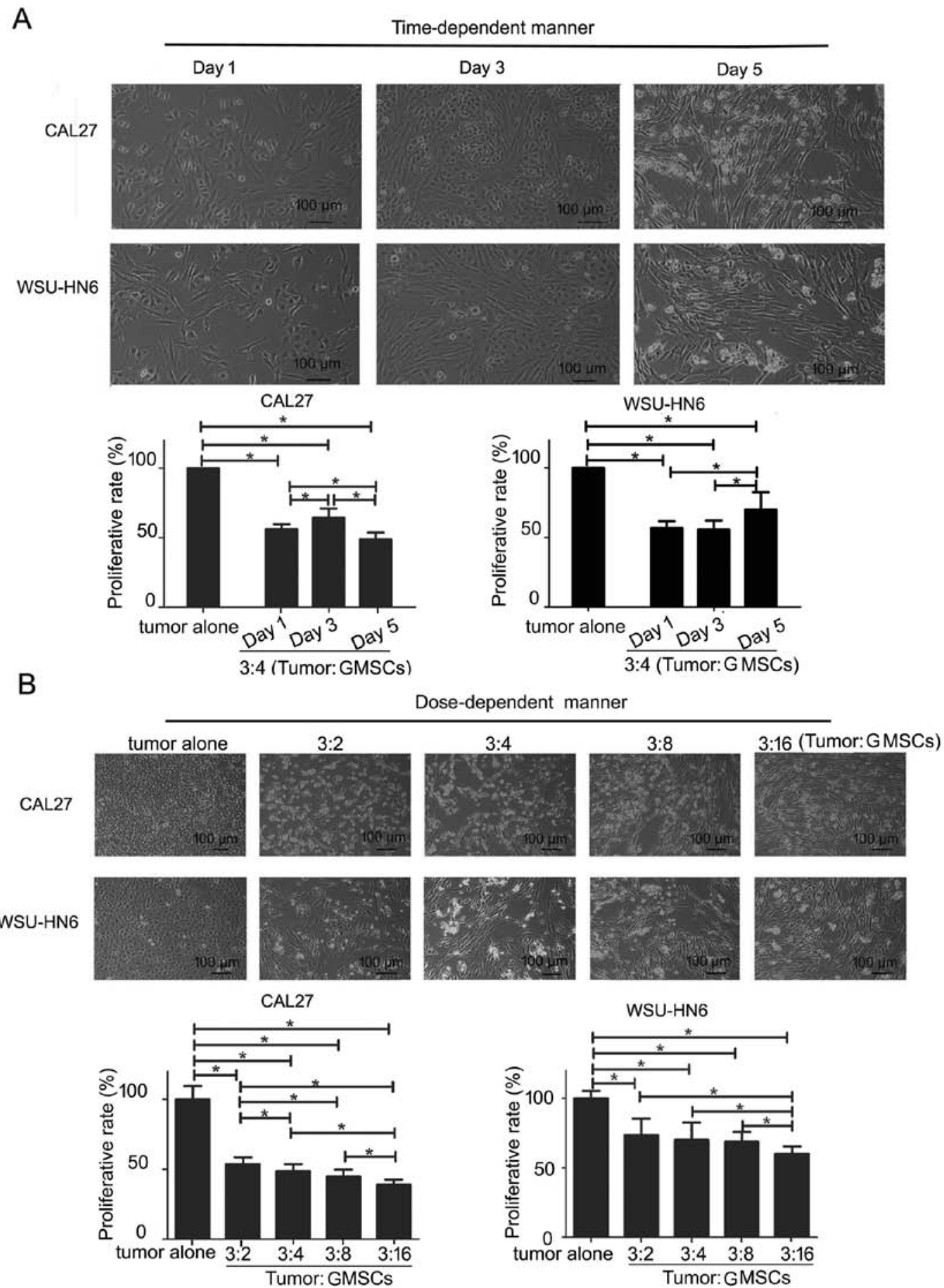

Figure 2. Direct oral cancer cell-GMSC contact co-culture. (A) Time-course assay: CAL27 and WSU-HN6 were treated with GMSCs (tumor:GMSCs 3:4) for 1, 3 and 5 days. (B) Dose-finding assay: CAL27 and WSU-HN6 were treated with GMSCs at indicated doses (tumor:GMSCs 3:2, 3:4, 3:8, 3:16) for 5 days. Cell status was photographed under light microscopy (bar, $100 \mu \mathrm{m}$ ), and cell viability was examined by MTT assay. Tumor alone was taken as controls. Error bars represent mean \pm SD. ${ }^{*} \mathrm{p}<0.05$.

Statistical analysis. Data are expressed as mean \pm standard deviation (SD). One-way ANOVA was used to evaluate the difference among groups. Significance was defined as a p-value of $<0.05$.

\section{Results}

Identification of GMSCs. We successfully generated GMSCs from normal gingival tissue. To identify the characteristics of
GMSCs, we used FITC- and PE-conjugated MSC biomarker antibodies to label GMSCs, and followed by flow cytometry assay. The results showed that GMSCs were positive for CD105, CD90, CD73, CD146, CD29 and STRO-1 and negative for CD34 and CD45 (Fig. 1A), which were consistent with previous studies $(14,15)$. To evaluate the multilineage differentiation abilities of normal GMSCs, osteogenic and adipogenic capacities were detected. Using osteoinductive condition, GMSCs formed mineralized nodules (Fig. 1B). 

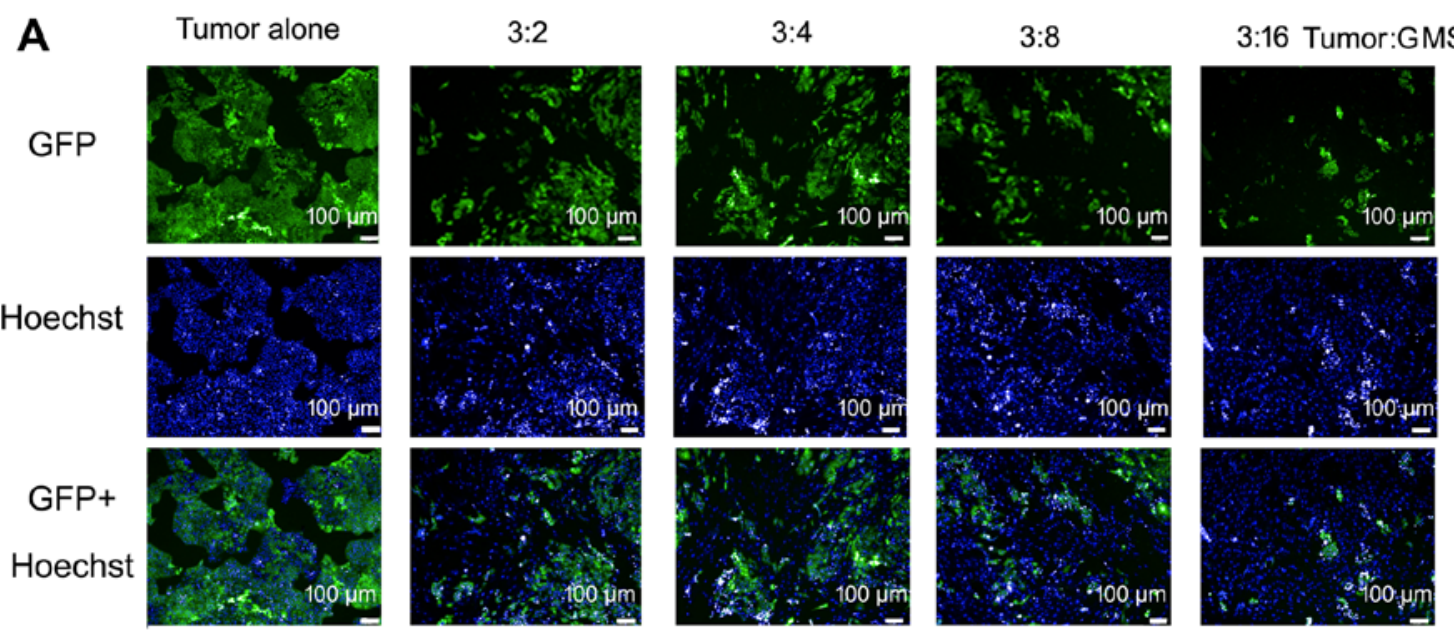

\section{B}
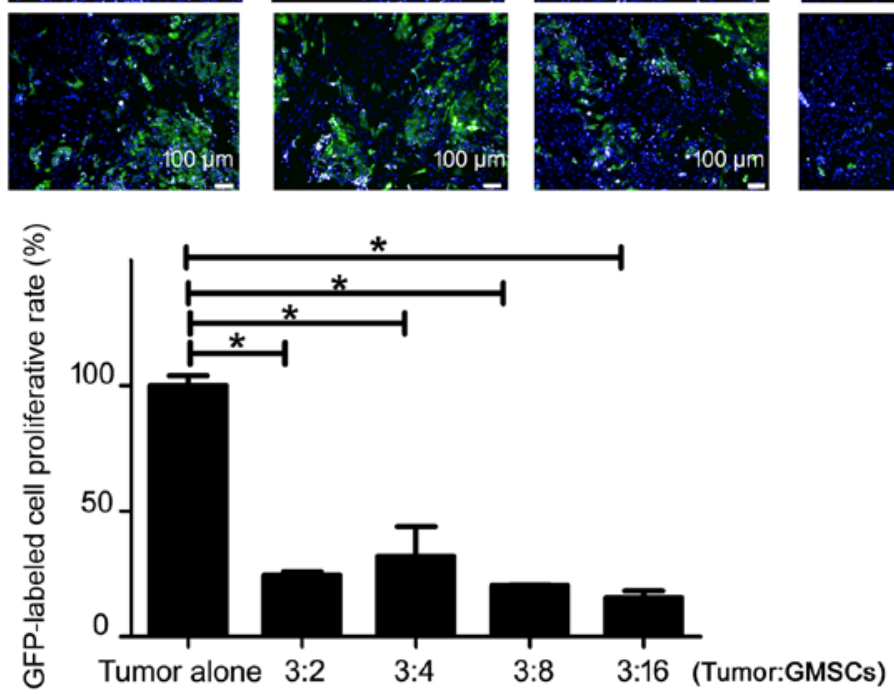

Figure 3. Direct GMSCs and GFP-labeled CAL27 contact co-culture at indicated doses (tumor:GMSCs 3:2, 3:4, 3:8, 3:16) for 5 days. (A) GFP-labeled CAL27 cells (green color) were photographed under a fluorescence microscope (bar, $100 \mu \mathrm{m}$ ). The nucleus (blue color) was stained by Hoechst 33342 . (B) The percentage of the number of GFP-labeled tumor cells was calculated. Tumor alone was taken as controls. Error bars represent mean $\pm \mathrm{SD}$. ${ }^{*} \mathrm{p}<0.05$.

Under adiogenic induction, GMSCs formed oil droplets (Fig. 1B). These properties of GMSCs are in line with the characteristics of MSCs $(14,15)$.

GMSCs inhibit oral cancer cell growth. In a time-course assay, when CAL27 and WSU-HN6 were treated with GMSCs for 5 days, the morphology of the two tumor cells did not change obviously at days 1 and 3, with a huge changed at day 5. Most tumor cells were suspended and shrank at day 5 (Fig. 2). MTT assay showed that GMSCs could cause a decrease in a number of GMSCs-CAL27 (WSU-HN6) co-culture at days 1,3 and 5 (p<0.05, Fig. 2A). On day 5, the inhibitory effect of GMSCs was in a dose-course (p $<0.05$, Fig. 2B). The total living cell number of GMSCs-CAL27 and GMSCs-WSU-HN6 co-culture was even lower than of the number in tumor alone group. Compared with tumor alone, at any dose of GMSCs exerted inhibitory proliferative effect on CAL27 at day 5 (p<0.05, Fig. 3).

To understand whether cell-cell direct contact can affect the growth inhibitory effect of GMSCs, we performed tumor cellGMSC indirect co-culture via Transwell system. Similar to the results of 5-day direct co-culture, GMSCs dose-dependently showed significant inhibitory effect compared with tumor alone ( $\mathrm{p}<0.05$, Fig. 4A). CAL27 and WSU-HN6 exhibited a decrease in the number of cells $(\mathrm{p}<0.05$, Fig. $4 \mathrm{~A})$. The results indicated that soluble factors released from GMSCs played an important role in the GMSC-mediated oral cancer cell proliferation.
To confirm this opinion, we generated conditioned medium, and used it to treat tumor cells. Similar to the results of direct co-culture and indirect co-culture via Transwell assay, conditioned medium from GMSCs could significantly suppress the growth of CAL27 and WSU-HN6 dose- and time-dependently $(\mathrm{p}<0.05)$. Furthermore, conditioned medium of GMSCs showed greater inhibitory effects on tumor cell lines than GMSCs in direct and indirect co-culture systems (Fig. 4B). In addition, we found an apparent discrepancy in the number of tumor cells at day 3 between Transwell and conditioned medium (Fig. 4A and B).

To eliminate the effect of serum consumption during generation of conditioned medium from GMSCs, we added extra fresh serum $(0,2,5$ and $10 \%)$ to conditioned medium (equivalent to the ratio of tumor:GMSCs was 3:16). The freshly-made conditioned media was used to evaluate the growth inhibitory effect of GMSCs $(\mathrm{p}<0.05)$. Similarly, serum concentration did not increase with the growth of tumor cells (Fig. 4C). These results raised the possibility that the anti-proliferation effect of GMSCs was not due to the serum deprivation.

Conditioned medium from GMSCs induces apoptosis of oral cancer cell lines. To investigate whether GMSCs inhibit the proliferation of oral cancer cell lines through apoptosis, we evaluated the effect of conditioned medium on oral cancer cells. At 24 and $48 \mathrm{~h}$ post-treatment by 5 -day conditioned 
A

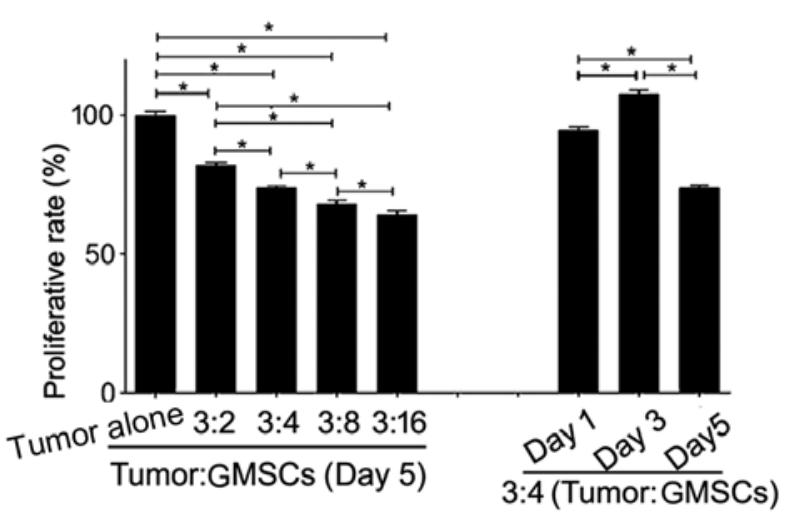

Transwell

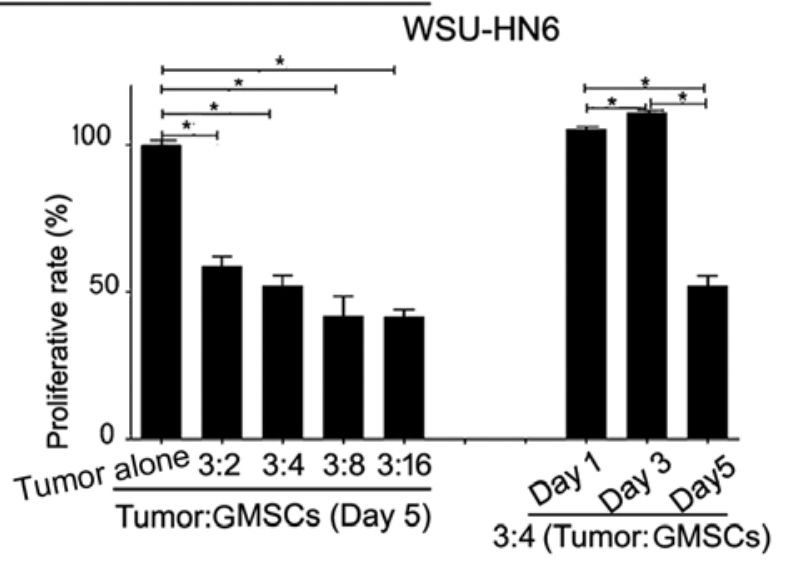

B

Conditioned medium

\section{CAL27}

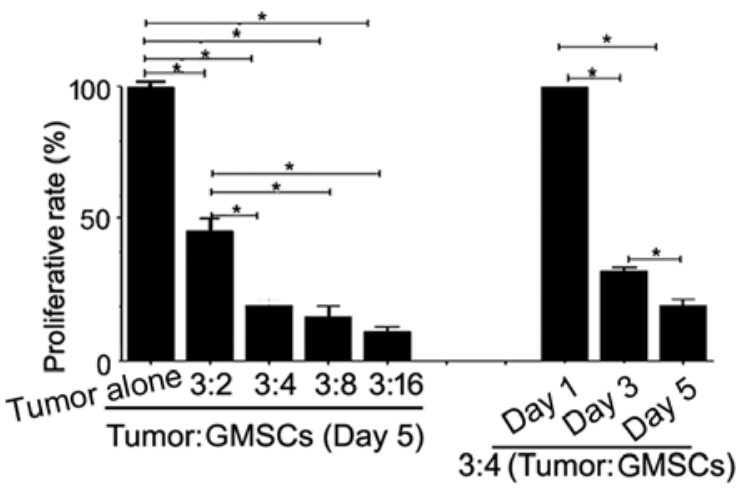

WSU-HN6

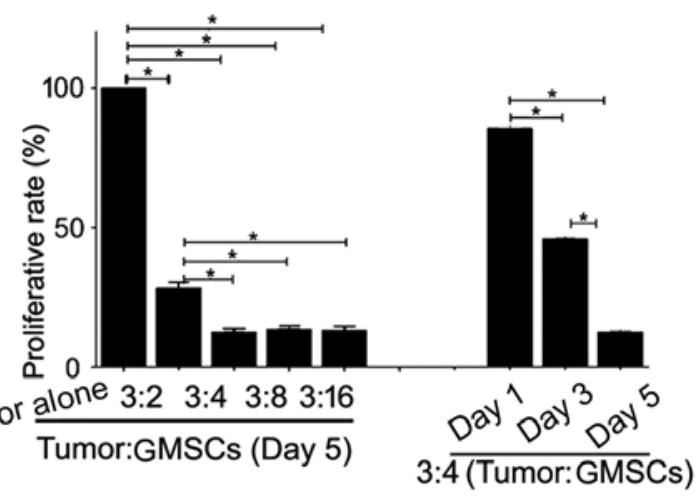

C Exclusive serum consumption assay
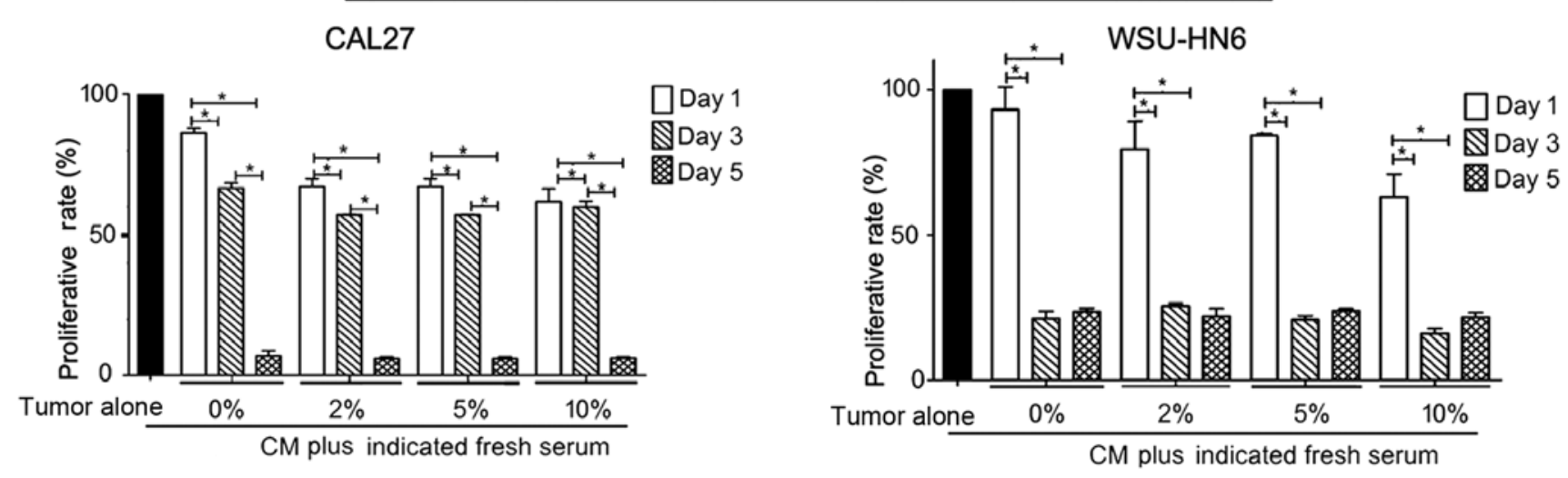

Figure 4. Effects of GMSCs on oral cancer cell growth through indirect cell-cell contact co-culture and conditioned medium from GMSCs with/without fresh serum supplement. CAL27 and WSU-HN6 were treated with indicated doses of GMSCs for various times via Transwell system (A) or conditioned medium from GMSCs without additional serum supplement (B) or with additional serum supplement (C). Cell viability was determined by MTT assay. Tumor alone was the control. Error bars represent mean \pm SD of three independent experiments. ${ }^{*} \mathrm{p}<0.05$. CM, conditioned medium.

medium (equivalent to the ratio of tumor:GMSCs was 3:4), CAL27 and WSU-HN6 were harvested for morphology evaluation and apoptosis assay. As shown in Fig. 5A, morphology checked by a microscope displayed that the numbers of tumor cells were partially suspended, and shrunken $48 \mathrm{~h}$ post-treatment. The results indicated that tumor cells may have undergone apoptosis. Therefore, apoptosis assay was performed, and the results showed that the percentage of apoptotic cells [early apoptotic (Annexin $\left.\mathrm{V}-\mathrm{FITC}^{+} / \mathrm{PI}^{-}\right)+$late apoptotic cells (Annexin V-FITC ${ }^{+} / \mathrm{PI}^{+}$) cells)] increased from $2.12 \pm 1.87$ (Ctrl) to $15.12 \pm 4.07 \%$ (treatment, $\mathrm{p}<0.05$ ) in CAL27, and from $2.04 \pm 0.58$ (Ctrl) to $38.90 \pm 6.53 \%$ (treatment, $\mathrm{p}<0.05$ ) in WSU-HN6 (Fig. 5B).

Detection of the concentration of cytokines in conditioned medium of GMSCs. To determine the concentration of 
A

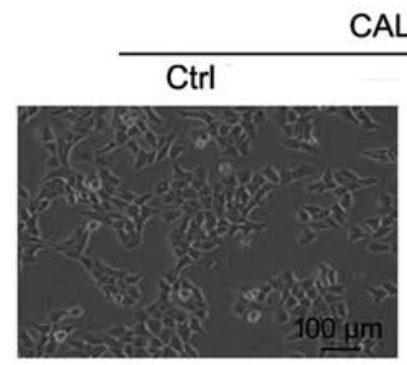

$24 \mathrm{~h}$

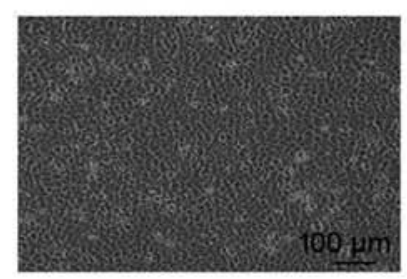

CAL27
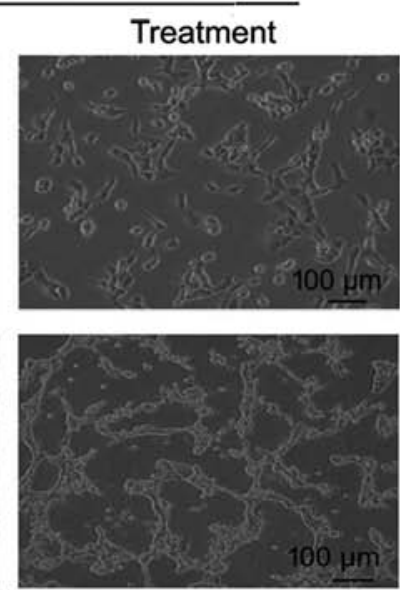
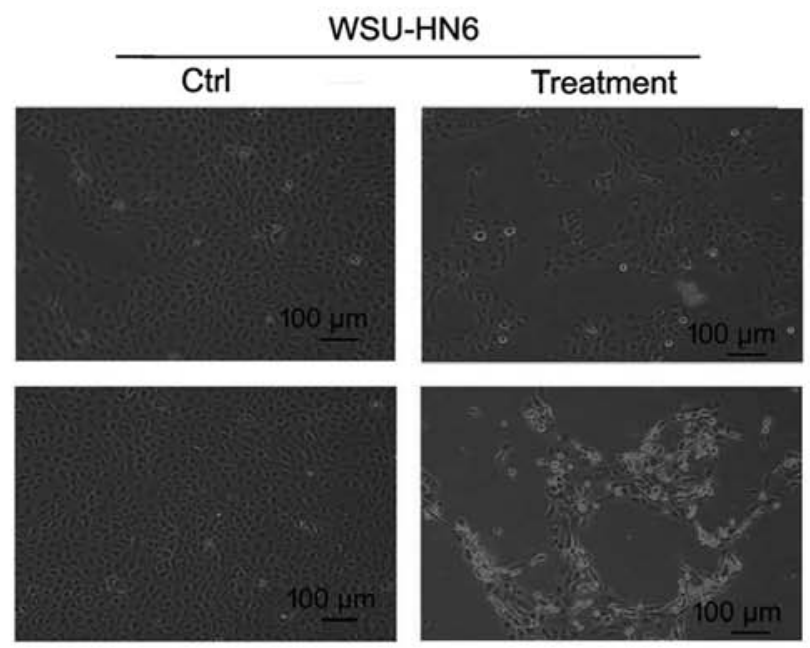

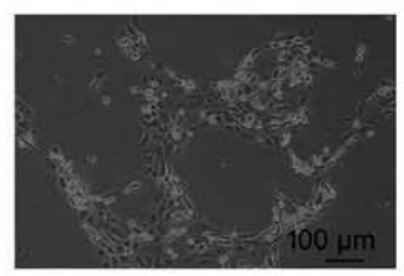

CAL27

B

Ctrl
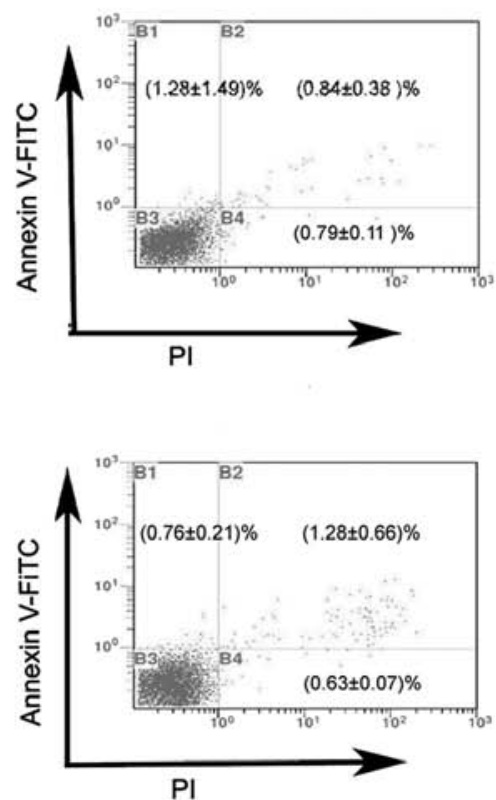

Treatment
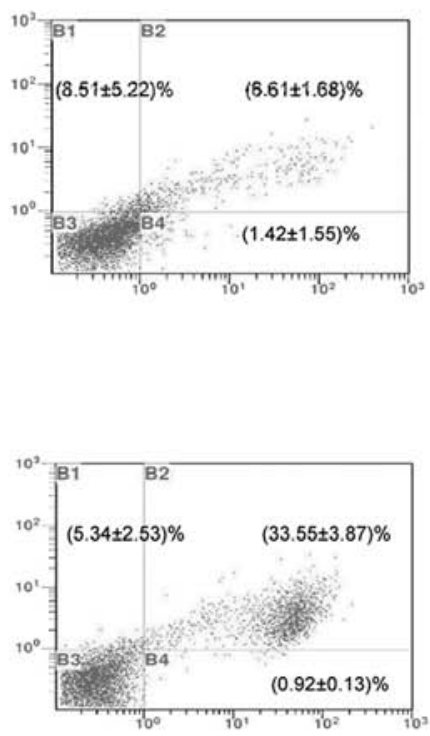

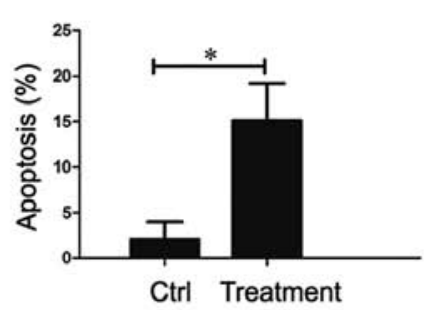

WSU-HN6

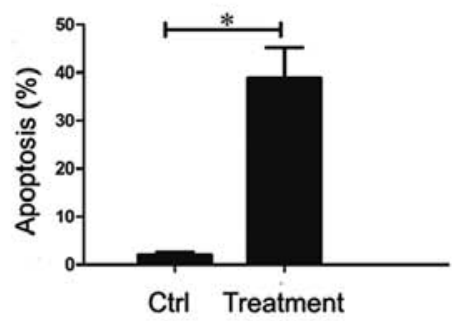

Figure 5. Apoptosis of oral cancer cells was induced by conditioned medium (equivalent to the ratio of tumor:GMSCs was 3:4) for 5 days. (A) Cell morphology was recorded under a light microscope at the indicated time points. (B) CAL27 and WSU-HN6 cells were treated with conditioned medium for $48 \mathrm{~h}$. Apoptosis was detected by flow cytometry after Annexin V-FITC/PI staining, and the percentage of apoptotic cells (=B1+B2) was quantified. Tumor alone was as control. Error bars represent mean $\pm \mathrm{SD}$ of three independent experiments. ${ }^{\mathrm{p}} \mathrm{p}<0.05$.

cytokines in conditioned medium of GMSCs, we carried out custom cytokine array. Results showed that, among these cytokines, only IL-6, IL-8 and GM-CSF's concentration was in the range of their biological activities (Table I). Based on previous studies, concentration of IFN- $\gamma$, which was out of the range of biological activities, indicated that IFN- $\gamma$ might not play an important role in tumor cell proliferation or play a synergic effect with other cytokines $(4,16)$. Therefore, we chose IL-6, IL-8, GM-CSF and IFN- $\gamma$ to reconstruct the artificial conditioned medium, and then treated the tumor cells with the artificial conditioned medium. The results showed that some cytokine combinations (GM-CSF+IFN- $\gamma$ and IFN- $\gamma+$ IL-6+IL-8 in CAL27 groups and GM-CSF+IL-6, IFN- $\gamma+$ IL- 8 and GM-CSF+IL-8+IFN- $\gamma$ in WSU-HN6 groups) exhibited a significant anti-proliferative effect on CAL27 or WSU-HN6 compared with corresponding tumor alone
Table I. Cytokine concentration in the conditioned medium of GMSCs (pg/ml).

\section{Cytokine}

Concentration

\section{GM-CSF}

850.55

IFN- $\gamma$

11.56

IL-10

11.9

IL-2

9.72

IL-4

15.2

IL-6

11,275

IL-8

3,491

CCL-2

12,750

TNF- $\alpha$

15.6

VEGF-A 

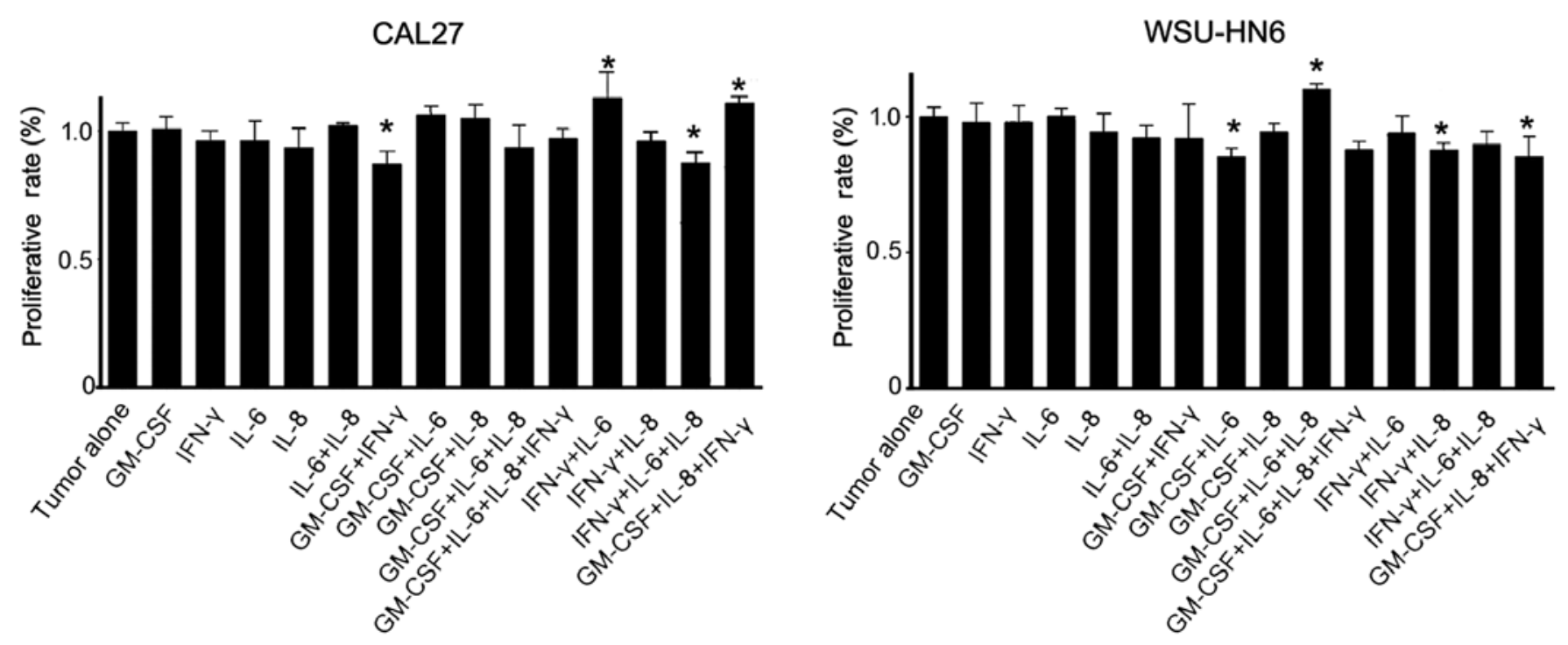

Figure 6. CAL27 and WSU-HN6 were treated with reconstructed artificial conditioned medium, which has the same concentrations as those in conditioned medium for GMSCs cultured for 5 days. Cell viability was examined by MTT assay. Tumor alone was the control. Error bars represent mean \pm SD of three independent experiments. ${ }^{*} \mathrm{p}<0.05$.

A

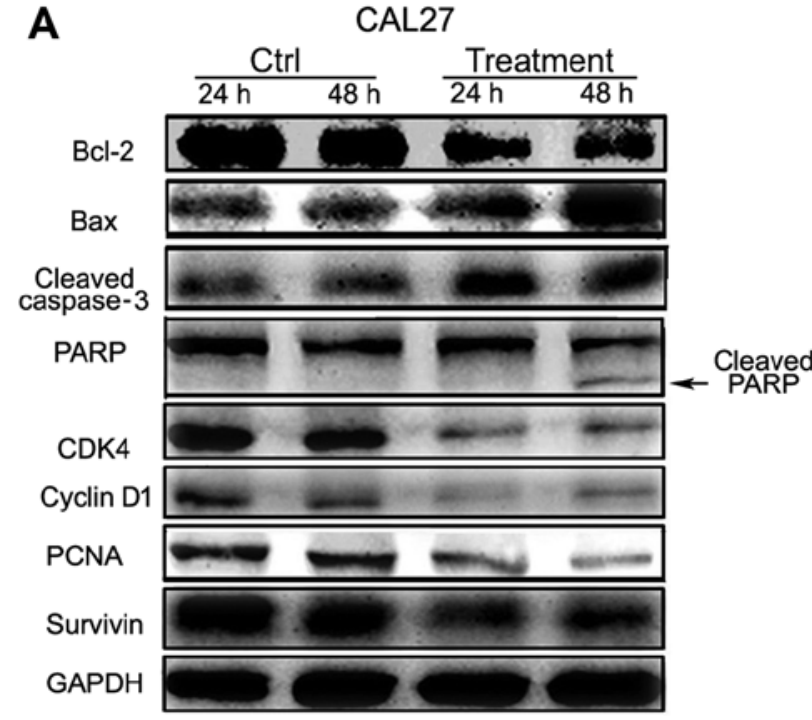

B

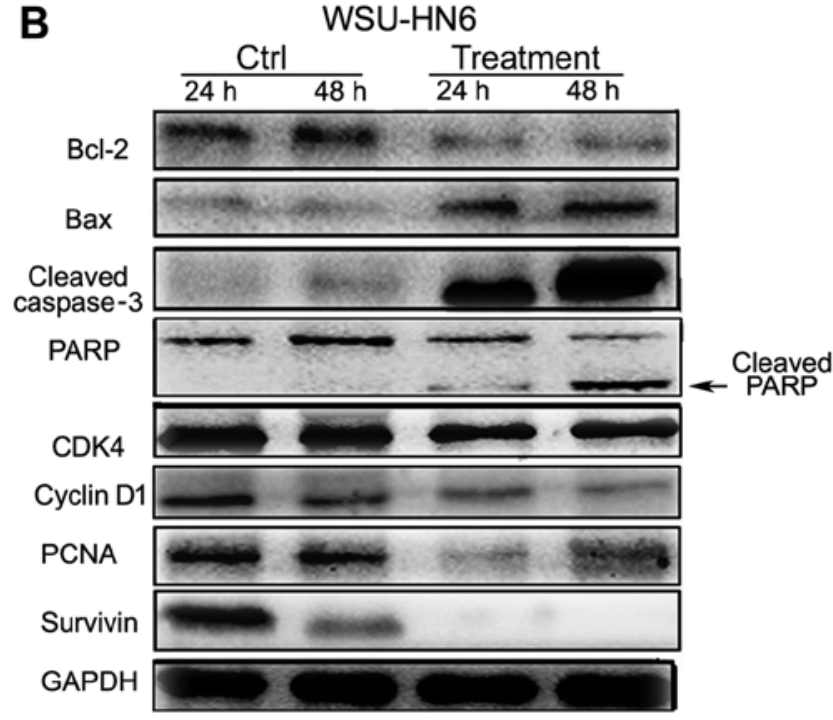

Figure 7. GMSCs inhibit oral cancer cell growth and promote apoptosis of oral cancer cell lines. CAL27 and WSU-HN6 were treated with conditioned medium derived from GMSCs cultured for 5 days (equivalent to the ratio of tumor:GMSCs was 3:4 in co-culture system) for 24 and $48 \mathrm{~h}$, and then subjected to western blotting for detection of Bcl-2, Bax, cleaved caspase-3, PARP, CDK4, cyclin D1, PCNA and survivin expression.

$(\mathrm{p}<0.05)$. In addition, IFN- $\gamma+\mathrm{IL}-8+\mathrm{GM}-\mathrm{CSF}-$, IFN- $\gamma+\mathrm{IL}-6-$ treated CAL27 or GM-CSF+IL-6+IL-8-treated WSU-HN6 showed slightly pro-proliferative effects ( $<<0.05$, Fig. 6). However, compared with the inhibitory effect of GMSCs or GMSCs-CM on the growth of the two oral cancer cell lines, the constructed cytokine mixture only exerted a very slight anti-proliferation effect on oral cancer cells. The results indicated that there may be unknown soluble factors, which inhibit the growth of oral cancer cell lines in the conditioned medium from GMSCs.

GMSCs downregulate the proliferation-related gene expression and upregulate apoptosis-related gene expression. To assess the molecular changes regarding proliferation and apoptosis during the process of GMSCs inhibiting the growth of tumor cells, CAL27 and WSU-HN6 were treated with conditioned medium (equivalent to the ratio of tumor:GMSCs referred to as 3:4) for 24 and $48 \mathrm{~h}$, the cells were examined by western blotting for detection of Bcl-2, Bax, cleaved caspase-3, PARP, CDK4, cyclin D1, PCNA and survivin. The results showed that a decrease in $\mathrm{Bcl}-2$ and a gradual increase in Bax, cleaved caspase-3 and cleaved PARP in CAL27 and WSU-HN6 from 24 to $48 \mathrm{~h}$ (Fig. 7). The expression of positive cell proliferative markers, such as CDK4, cyclin D1, PCNA and survivin were markedly downregulated compared to control groups (Fig. 7). 
A

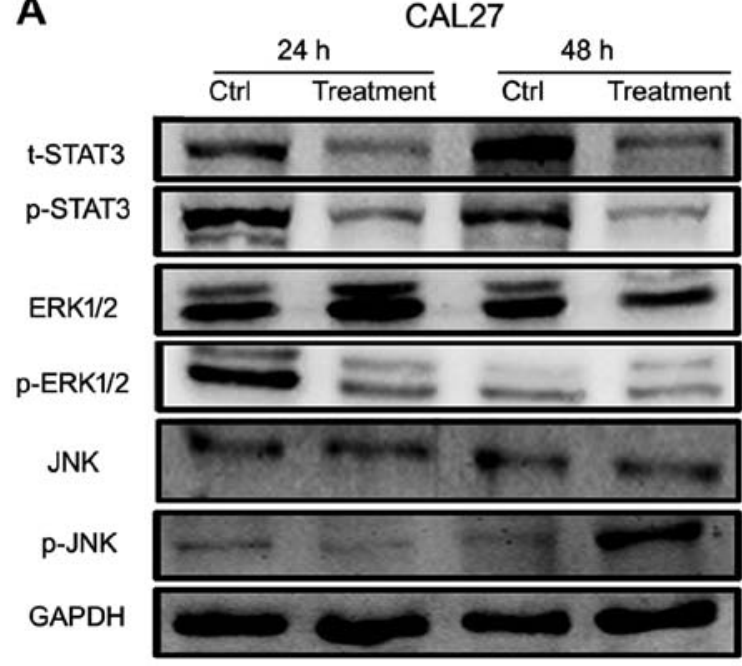

B

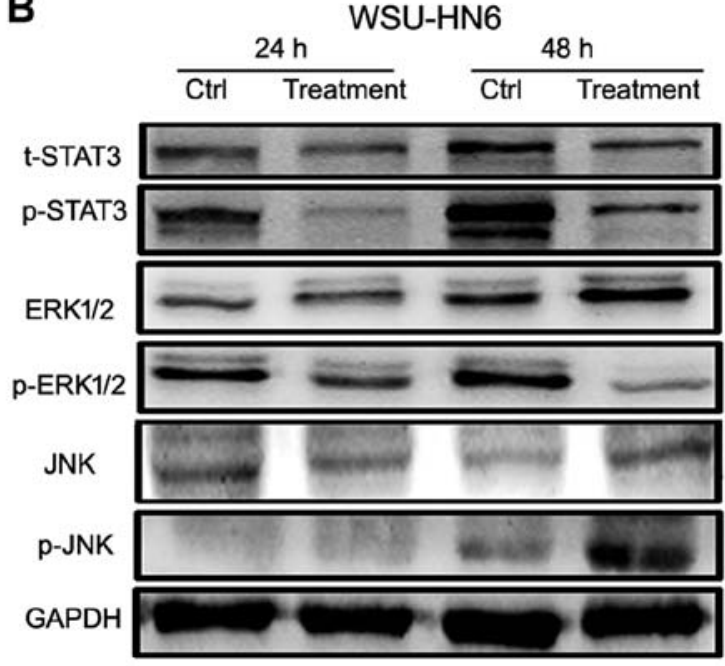

Figure 8. GMSCs promote apoptosis of oral cancer cells partially through JNK signaling pathway. CAL27 and WSU-HN6 were treated with conditioned medium of GMSCs (equivalent to the ratio of tumor:GMSCs was 3:4 in co-culture system for 5 days) for 24 and 48 h, and then subjected to western blotting for detection of expression of signal transduction pathways.

To determine which pathway is involved in the process of conditioned medium of GMSC-induced oral cancer cell growth inhibition, we detected proliferation-associated pathway including STAT3, JNK, ERK pathways by western blotting. We found that GMSCs-CM could activate the JNK pathway through phosphorylation of JNK (p-JNK), and inactivate ERK and STAT3 pathways through a decrease in p-ERK1/2 and p-STAT3 (Fig. 8).

JNK inhibitor blocks the growth inhibitory effect of GMSCs on oral cancer cells. To find whether pathway is involved in the process of GMSCs inhibiting the growth of tumor cells, we performed pathway inhibition assay. CAL27 or WSU-HN6 were pretreated with JNK inhibitor SP600125 for $1 \mathrm{~h}$, then treatment was continued with conditioned medium containing SP600125. At $24 \mathrm{~h}$ post-treatment, more apoptotic cells were found in conditioned medium group than SP600125 plus conditioned medium group by microscopy (Fig. 9A). MTT results showed that less viable cells were found in conditioned medium group than SP600125 plus conditioned medium group (Fig. 9A). Moreover, western blotting showed that SP600125 partly abrogated the expression of p-JNK, increased p-ERK1/2, Bcl-2, survivin expression and decreased Bax, cleaved caspase-3 expression (Fig. 9B).

GMSCs inhibit oral cancer cell growth in vivo. To investigate whether GMSCs have a similar growth inhibitory effect on oral cancer cells in vivo, we carried out an animal study. GMSCs and CAL27 were co-injected in nude mice. The volume of tumor was recorded every 4 days. We found that the volume of tumor in CAL27 plus GMSCs group was significantly smaller that in CAL27 alone group ( $\mathrm{p}<0.05$, Fig. 10). It suggested that GMSCs were able to inhibit the growth of CAL27 in vivo.

\section{Discussion}

In tumorigenesis, mutated epithelial cells interact with surrounding stromal cells participating in the establishment of tumor microenvironment, which is critically important for tumor development (17). Actually, unlike cancer cells, stromal cells in the tumor microenvironment are genetically stable and may be an attractive therapeutic target (18). As the precursor of most stromal cells in tumor microenvironment, MSCs gained extensive attention when evidence suggested tumor-associated MSCs present a distinct phenotype compared to MSCs derived from normal tissue (19). In adult tissue, the microenvironment protects the slow-cycling, self-renewal potential and undifferentiated state $(11,20)$. In tumor microenvironment, various inflammatory cytokines, chemokines, and growth factors can be secreted by tumor-associated MSCs, which can form the inflammatory environment and promote tumor progression (21). Many studies have shown that normal tissue-derived MSCs are capable of remodeling tumor microenvironment, rather than only target cancer cells. In our study, we firstly isolated MSCs from normal gingival tissues, and identified that GMSCs have heterogeneity to $\mathrm{STRO}-1^{+} / \mathrm{CD} 0^{+} / \mathrm{CD} 105^{+} /$ $\mathrm{CD}_{146}{ }^{+} / \mathrm{CD} 3^{+} / \mathrm{CD} 29^{+}$and CD34/CD $45^{-} \mathrm{MSCs}$, and have osteogenic and adipogenic capacities. The result is consistent with a previous study (13).

Next, we investigated the effect of GMSCs on oral cancer cell proliferation. To our surprise, by MTT and apoptosis assays, we found that GMSCs had strong growth inhibition in the two tumor cells not only by cell-cell direct contact, but also by cell-cell indirect contact via Transwell system. Our findings are consistent with some reports (22). Other contrary reports showed that MSCs could enhance the growth of several different tumors. The reason for this discrepancy is unknown, but it may be associated with the tropism of MSCs and differences in the tumor model, the dose or timing of MSCs applied, or other unknown reasons (23). So how to choose the source of MSCs to be applied to different tumors still needs to be explored. Our results indicate that using MSCs derived from the same tissue/organ region as the tumor cells can be an appropriate choice for cancer treatment. Our rationale is that normal MSCs can maintain the homeostasis of epithelial tissue. Once tumor occurs, homeostasis is broken, normal MSCs 
A
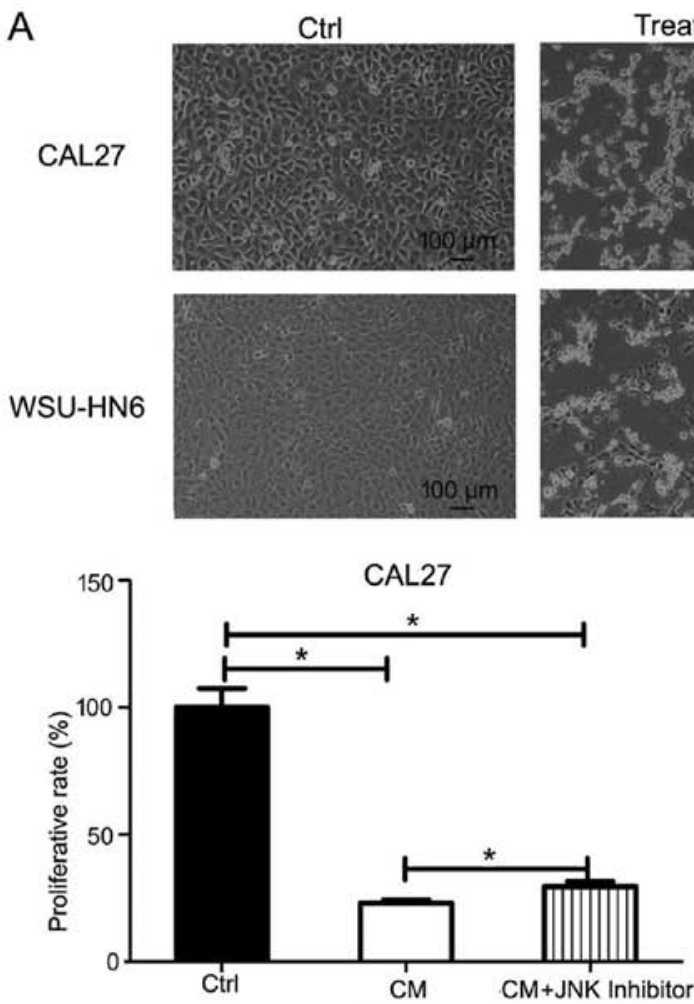

B

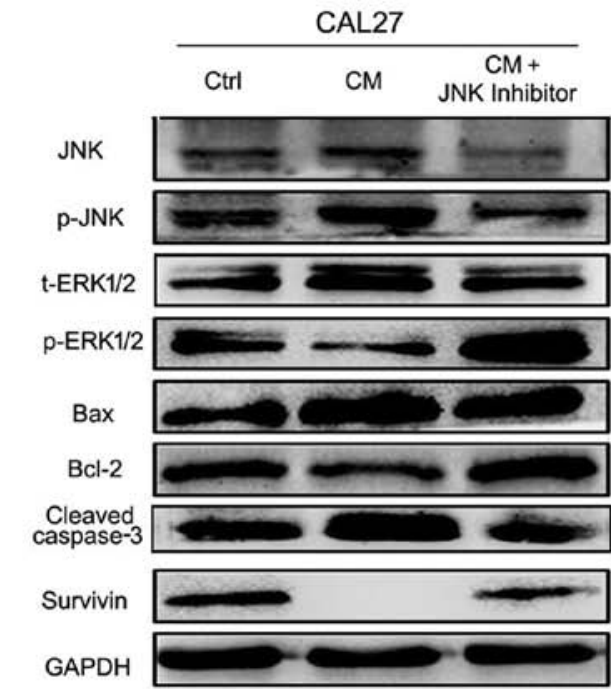

Treatment
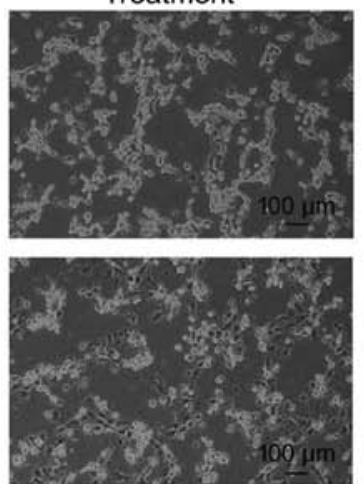

$\mathrm{CM}+\mathrm{JNK}$ Inhibitor
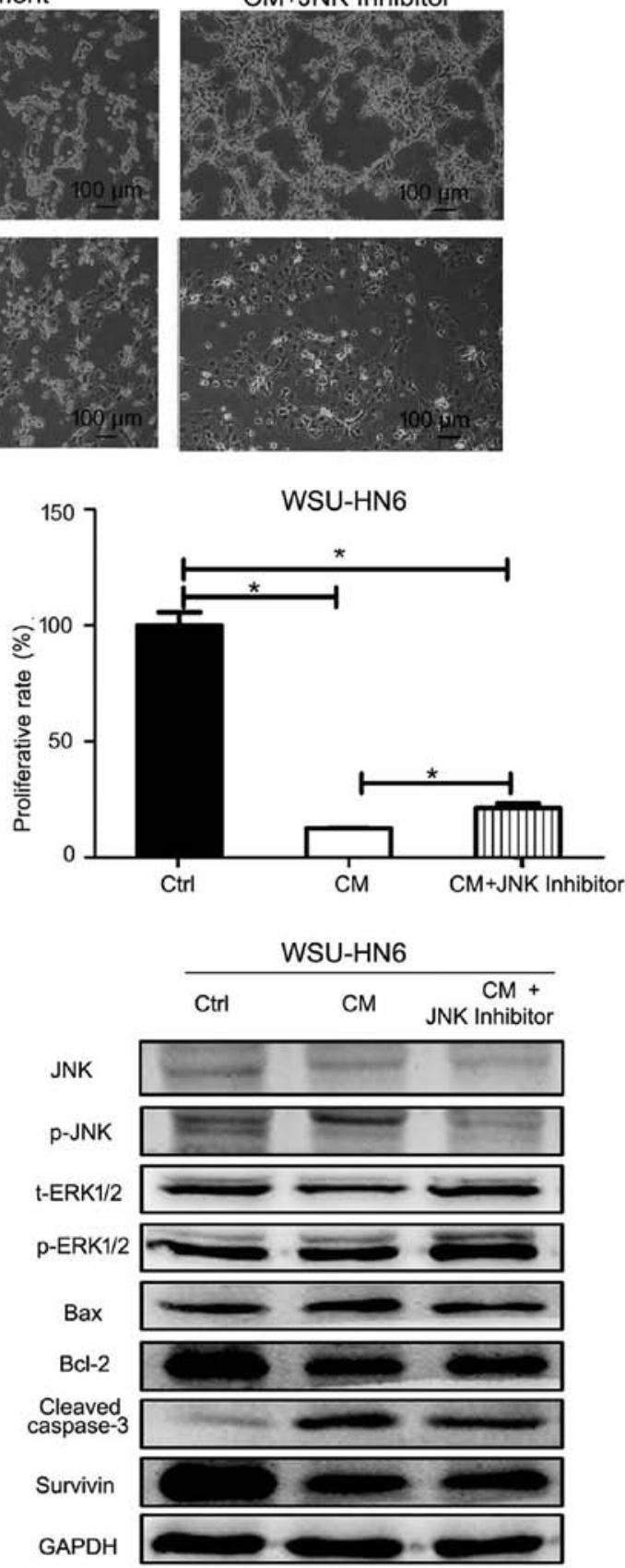

Figure 9. Effect of JNK inhibitor on apoptosis induced by GMSCs in oral cancer cells. (A) CAL27 and WSU-HN6 were treated with $10 \mathrm{~K}-\mathrm{CM}$ for $48 \mathrm{~h}$, cell morphology was recorded under a microscope (bar, $100 \mu \mathrm{m}$ ), and cell viability was determined by MTT assay. (B) Effect of JNK inhibitor on conditioned medium derived from GMSCs on the expression of p-JNK, p-ERK1/2, Bax, Bcl-2, cleaved caspase-3, survivin in CAL27 and WSU-HN6. Error bars represent mean \pm SD. ${ }^{*} p<0.05$.

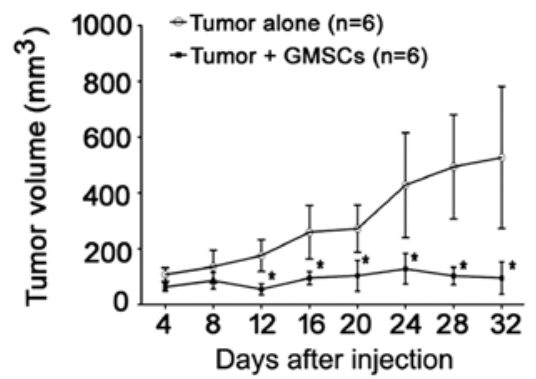

Figure 10. Inhibitory effect of GMSCs on CAL27 cells in vivo. CAL27 $\left(2 \times 10^{6}\right.$ cells) and GMSCs $\left(1 \times 10^{6}\right.$ cells) were co-injected into nude mice. The volume of tumor was recorded every 4 days. "p $<0.05$. surrounding tumor may become tumor-associated fibroblasts, which normal tissue microenvironment also changes to tumor microenvironment. Therefore, using normal MSCs from the same tissue/organ origin of tumor to treat tumor perhaps corrects the altered microenvironment by tumor, and exerts an anticancer effects.

In this study, we found that soluble factors secreted by GMSCs play an important role in GMSC-induced oral cancer growth inhibition. To confirm this result, we generated conditioned medium from GMSCs and found that conditioned medium had stronger growth inhibition than the above described cell-cell co-culture. We suspect that the stronger 
inhibition growth of conditioned medium may be associated with an insufficient amount of inhibitory cytokines at days 1 and 3 in a cell-cell co-culture and Transwell. Furthermore, this effect is dose- and time-dependent, and regardless of serum concentration in the conditioned medium. Our explanation is that at the beginning of cell-cell co-culture, the quantity of the soluble factors released from GMSCs is very low and not enough to inhibit oral cancer cell growth, even in turn promotes oral cancer cell growth, just like the day 3 data in co-culture system in this study. Along with the time extension, GMSCs secrete more and more soluble factors and gradually exert inhibitory effect on oral cancer cells $(2,22,24)$.

To further explore which factors play the main role in GMSC-induced oral cancer cell growth inhibition, we performed a custom cytokine array and found that IL-6, IL-8, GM-SCF in conditional medium of GMSCs was in the range of their biological activities. Our results were in line with some reports. Motaln et al (16) reported that IL-6, IL-8, CCL2 are detected in hMSCs/glioblastoma multiforme co-culture medium known to inhibit the growth of tumors. Kucerova et al (4) showed that inhibition of glioblastoma multiforme growth may be mediated by high cytokine levels produced by adiposederived MSCs, in particular high levels of interleukins (IL-1b, IL-1ra, IL-2, IL-4, IL-6, IL-8) combined with high levels of IFN- $\gamma$ and G-CSF. By construction of the artificial conditioned media with the same quantity of the above differentially expressed factors in the conditioned medium, we did not find similar anti-proliferation effect as GMSCs or GMSCs-CM in this study. The results indicate that there must be unknown soluble proteins, exosome or other unknown factor released from GMSCs, which are the main contributor(s) for GMSCinduced oral cancer cell growth inhibition.

Although we did not find the unknown main soluble factors, we found the pathway by which GMSCs exert the inhibitory growth effect of oral cancer cell lines. We carried out western blotting to detect the proteins including Bcl-2 (25), p-STAT3 (26), survivin (27), proliferation (ERK1/2) (28) and apoptosis [Bax (25), cleaved caspase-3 (29), JNK (30)] which are associated with proliferation, survival or apoptosis. The protein expression is in line with the observed phenomena in this study as well as the function of sthese proteins. Furthermore, by pathway inhibition assay, we found that JNK is involved in GMSC-induced oral cancer cell growth inhibition, JNK activity is important in the regulation of tumor cell proliferation. JNK inhibitor reversed the pro-apoptotic ability mediated by GMSCs through downregulation of Bax, cleaved caspase-3 and $\mathrm{p}-\mathrm{JNK}$, and upregulation of survivin and p-ERK1/2. It is suggested that modulation of JNK can promote the key factors in inducing apoptosis via Bax and Bcl-2. Ryu et al also reported that ASCs suppressed tumor growth via JAK1/JAK2 pathway (2), so it can be explained that GMSCs inhibit the growth of oral cancer cells not only by JNK pathway, but also other pathways.

To further confirm the growth inhibitory effect of GMSCs on oral cancer cells, we performed an animal study. Our results clearly showed that GMSCs were able to inhibit the growth of CAL27 in nude mice. Therefore, we deduce that GMSCs have a promising clinical potential in the management of oral cancer or oral epithelial dysplasia such as oral leukoplakia.
In conclusion, our results suggest that GMSCs can suppress oral cancer cell growth by activation of JNK signaling pathway. Unknown soluble factors released from GMSCs play a key role in GMSC-induced oral cancer cell growth inhibition. Further study is required to find which non-specified paracrine factor inhibits the proliferation of oral cancer cells and how to apply the potential of GMSC-mediated anticancer proliferation effect in clinic.

\section{Acknowledgements}

This study was supported by the National Natural Science Foundation of China (nos. 81441034 and 81172556) and Foundation of Capital Health Development (2014-2-4102 and 2011-4025-02).

\section{References}

1. Bernardo ME and Fibbe WE: Mesenchymal stromal cells: Sensors and switchers of inflammation. Cell Stem Cell 13: 392-402, 2013.

2. Ryu H, Oh JE, Rhee KJ, Baik SK, Kim J, Kang SJ, Sohn JH, Choi E, Shin HC, Kim YM, et al: Adipose tissue-derived mesenchymal stem cells cultured at high density express IFN- $\beta$ and suppress the growth of MCF-7 human breast cancer cells. Cancer Lett 352: 220-227, 2014

3. Zhu Y, Sun Z, Han Q, Liao L, Wang J, Bian C, Li J, Yan X, Liu Y, Shao C, et al: Human mesenchymal stem cells inhibit cancer cell proliferation by secreting DKK-1. Leukemia 23: 925-933, 2009.

4. Kucerova L, Matuskova M, Hlubinova K, Altanerova V and Altaner C: Tumor cell behaviour modulation by mesenchymal stromal cells. Mol Cancer 9: 129, 2010.

5. Song N, Gao L, Qiu H, Huang C, Cheng H, Zhou H, Lv S, Chen L and Wang J: Mouse bone marrow-derived mesenchymal stem cells inhibit leukemia/lymphoma cell proliferation in vitro and in a mouse model of allogeneic bone marrow transplant. Int J Mol Med 36: 139-149, 2015.

6. Huang WH, Chang MC, Tsai KS, Hung MC, Chen HL and Hung SC: Mesenchymal stem cells promote growth and angiogenesis of tumors in mice. Oncogene 32: 4343-4354, 2013.

7. Egusa H, Okita K, Kayashima H, Yu G, Fukuyasu S, Saeki M, Matsumoto T, Yamanaka S and Yatani H: Gingival fibroblasts as a promising source of induced pluripotent stem cells. PLoS One 5: e12743, 2010

8. Liu J, Yu F, Sun Y, Jiang B, Zhang W, Yang J, Xu GT, Liang A and Liu S: Concise reviews: Characteristics and potential applications of human dental tissue-derived mesenchymal stem cells. Stem Cells 33: 627-638, 2015.

9. Xu X, Chen C, Akiyama K, Chai Y, Le AD, Wang Z and Shi S: Gingivae contain neural-crest- and mesoderm-derived mesenchymal stem cells. J Dent Res 92: 825-832, 2013.

10. Tomar GB, Srivastava RK, Gupta N, Barhanpurkar AP, Pote ST, Jhaveri HM, Mishra GC and Wani MR: Human gingiva-derived mesenchymal stem cells are superior to bone marrow-derived mesenchymal stem cells for cell therapy in regenerative medicine. Biochem Biophys Res Commun 393: 377-383, 2010.

11. Wang F, Yu M, Yan X, Wen Y, Zeng Q, Yue W, Yang P and Pei X: Gingiva-derived mesenchymal stem cell-mediated therapeutic approach for bone tissue regeneration. Stem Cells Dev 20: 2093-2102, 2011.

12. Petersen PE: Oral cancer prevention and control - the approach of the World Health Organization. Oral Oncol 45: 454-460, 2009.

13. Zhang Z, Han Y, Song J, Luo R, Jin X, Mu D, Su S, Ji X, Ren YF and Liu H: Interferon- $\gamma$ regulates the function of mesenchymal stem cells from oral lichen planus via indoleamine 2,3-dioxygenase activity. J Oral Pathol Med 44: 15-27, 2015.

14. Hung BP, Hutton DL, Kozielski KL, Bishop CJ, Naved B, Green JJ, Caplan AI, Gimble JM, Dorafshar AH and Grayson WL: Platelet-derived growth factor BB enhances osteogenesis of adipose-derived but not bone marrow-derived mesenchymal stromal/stem cells. Stem Cells 33: 2773-2784, 2015.

15. Lei M, Li K, Li B, Gao LN, Chen FM and Jin Y: Mesenchymal stem cell characteristics of dental pulp and periodontal ligament stem cells after in vivo transplantation. Biomaterials 35: 6332-6343, 2014 
16. Motaln H, Gruden K, Hren M, Schichor C, Primon M, Rotter A and Lah TT: Human mesenchymal stem cells exploit the immune response mediating chemokines to impact the phenotype of glioblastoma. Cell Transplant 21: 1529-1545, 2012.

17. Thiery JP: Epithelial-mesenchymal transitions in tumour progression. Nat Rev Cancer 2: 442-454, 2002.

18. Quail DF and Joyce JA: Microenvironmental regulation of tumor progression and metastasis. Nat Med 19: 1423-1437, 2013.

19. Liu R, Wei S, Chen J and Xu S: Mesenchymal stem cells in lung cancer tumor microenvironment: Their biological properties, influence on tumor growth and therapeutic implications. Cancer Lett 353: 145-152, 2014.

20. Urbanek K, Cesselli D, Rota M, Nascimbene A, De Angelis A, Hosoda T, Bearzi C, Boni A, Bolli R, Kajstura J, et al: Stem cell niches in the adult mouse heart. Proc Natl Acad Sci USA 103 9226-9231, 2006.

21. Sun Z, Wang S and Zhao RC: The roles of mesenchymal stem cells in tumor inflammatory microenvironment. J Hematol Oncol 7: 14, 2014

22. Qiao L, Xu ZL, Zhao TJ, Ye LH and Zhang XD: Dkk-1 secreted by mesenchymal stem cells inhibits growth of breast cancer cells via depression of Wnt signalling. Cancer Lett 269: 67-77, 2008.

23. Klopp AH, Gupta A, Spaeth E, Andreeff M and Marini F III: Concise review: Dissecting a discrepancy in the literature: do mesenchymal stem cells support or suppress tumor growth? Stem Cells 29: 11-19, 2011.
24. Qiao L, Xu Z, Zhao T, Zhao Z, Shi M, Zhao RC, Ye L and Zhang X: Suppression of tumorigenesis by human mesenchymal stem cells in a hepatoma model. Cell Res 18: 500-507, 2008.

25. Um HD: Bcl-2 family proteins as regulators of cancer cell invasion and metastasis: A review focusing on mitochondrial respiration and reactive oxygen species. Oncotarget 7: 5193-5203, 2016.

26. Su TH, Shiau CW, Jao P, Liu CH, Liu CJ, Tai WT, Jeng YM, Yang HC, Tseng TC, Huang HP, et al: Sorafenib and its derivative SC-1 exhibit antifibrotic effects through signal transducer and activator of transcription 3 inhibition. Proc Natl Acad Sci USA 112: 7243-7248, 2015.

27. Li Y, Liu D, Zhou Y, Li Y, Xie J, Lee RJ, Cai Y and Teng L: Silencing of survivin expression leads to reduced proliferation and cell cycle arrest in cancer cells. J Cancer 6: 1187-1194, 2015.

28. Sheridan C, Brumatti G, Elgendy M, Brunet M and Martin SJ: An ERK-dependent pathway to Noxa expression regulates apoptosis by platinum-based chemotherapeutic drugs. Oncogene 29: 64286441, 2010.

29. Wier EM, Fu K, Hodgson A, Sun X and Wan F: Caspase-3 cleaved p65 fragment dampens NF- $\kappa \mathrm{B}$-mediated anti-apoptotic transcription by interfering with the p65/RPS3 interaction. FEBS Lett 589: 3581-3587, 2015.

30. Bogoyevitch MA and Kobe B: Uses for JNK: The many and varied substrates of the c-Jun N-terminal kinases. Microbiol Mol Biol Rev 70: 1061-1095, 2006. 